\title{
Compton Scattering Program Studying Nucleon Polarizabilities
}

\author{
Philippe Martel ${ }^{1,2, \star}$, Maik Biroth $^{1}$, Cristina Collicott ${ }^{1,3,4,5}$, Dilli Paudyal ${ }^{6}$, and Ali Rajabi ${ }^{7}$ \\ on behalf of the $\mathrm{A} 2$ collaboration at MAMI \\ ${ }^{1}$ Institut für Kernphysik, Universität Mainz, D-55099 Mainz, Germany \\ ${ }^{2}$ Department of Physics, Mount Allison University, Sackville, New Brunswick E4L 1E6, Canada \\ ${ }^{3}$ Department of Physics, The George Washington University, Washington, D.C. 20052, USA \\ ${ }^{4}$ Department of Physics and Atmospheric Science, Dalhousie University, Halifax, Nova Scotia B3H 4R2, Canada \\ ${ }^{5}$ Department of Astronomy and Physics, Saint Mary's University, Halifax, Nova Scotia B3H 3C3, Canada \\ ${ }^{6}$ Department of Physics, University of Regina, Regina, Saskatchewan S4S OA2, Canada \\ ${ }^{7}$ Department of Physics, University of Massachusetts Amherst, Amherst, Massachusetts 01003, USA
}

\begin{abstract}
The A2 collaboration at the Mainz Microtron has undertaken a program of Compton scattering experiments with the goal of extracting the proton and, in the future, neutron polarizabilities. These are fundamental parameters that describe the response of the nucleon to an electromagnetic field. An extraction of these parameters will provide a crucial test of QCD theories. This talk presented the concept and status of this program, including some preliminary results.
\end{abstract}

\section{Introduction}

Nuclear Compton scattering, the interaction of an electromagnetic wave with a nucleon, can be described by a set of effective Hamiltonians. At lowest order this interaction is solely dependent on the mass, $m$, and electric charge, $e$, of the nucleon through

$$
H_{\mathrm{eff}}^{(0)}=\frac{\vec{\pi}^{2}}{2 m}+e \phi,
$$

where $\vec{\pi}=\vec{p}-e \vec{A}$. At the next order, the anomalous magnetic moment, $\kappa$, becomes relevant in

$$
H_{\mathrm{eff}}^{(1)}=-\frac{e(1+\kappa)}{2 m} \vec{\sigma} \cdot \vec{H}-\frac{e(1+2 \kappa)}{8 m^{2}} \vec{\sigma} \cdot[\vec{E} \times \vec{\pi}-\vec{\pi} \times \vec{E}]
$$

These first two orders encompass what are called the 'Born' terms, describing a point-like charged particle without internal structure.

\subsection{Scalar Polarizabilities}

As one goes to higher energies, the internal structure of the nucleon becomes evident by

$$
H_{\mathrm{eff}}^{(2)}=-4 \pi\left[\frac{1}{2} \alpha_{E 1} \vec{E}^{2}+\frac{1}{2} \beta_{M 1} \vec{H}^{2}\right],
$$

where $\alpha_{E 1}$ and $\beta_{M 1}$, so called scalar polarizabilities, represent the response of the nucleon to an applied electric or magnetic field, respectively. These effects can be visualized by imagining the nucleon being placed in a static field, as shown in Fig. 1. The application of an elec-

\footnotetext{
${ }^{\star}$ Speaker - e-mail: martel@uni-mainz.de
}
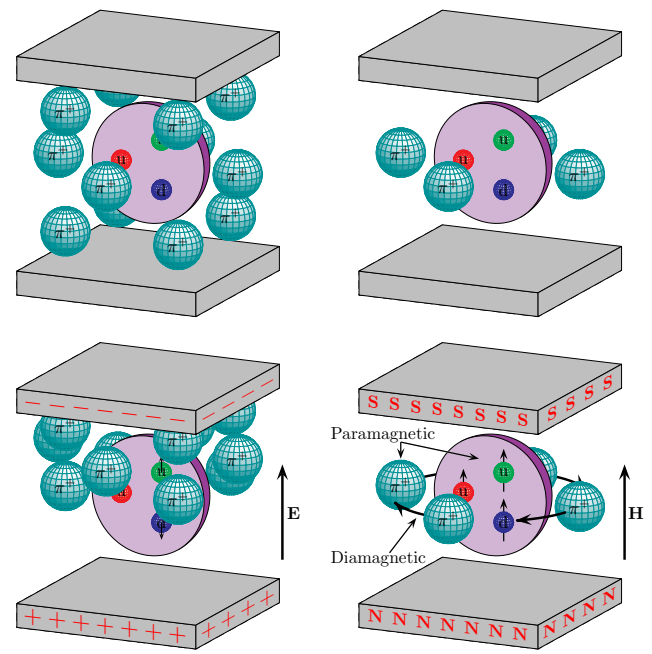

Figure 1. Simplistic visual representation of the response of a nucleon to an applied electric (left) or magnetic (right) field.

tric field causes a current within the pion cloud, effectively 'stretching' the nucleon. The application of a magnetic field causes both a diamagnetic moment within the pion cloud and a paramagnetic moment in the constituent quarks. Though the scalar polarizabilities for the proton have been determined through various Compton scattering experiments [1], some recent studies [2][3] have caused the Particle Data Group (PDG) to adjust the pre- 
viously quoted numbers [4]. Additionally, there is still interest in reducing the errors on these two factors, with the current values of $\alpha_{E 1}=(11.2 \pm 0.4) \times 10^{-4} \mathrm{fm}^{3}$ and $\beta_{M 1}=(2.5 \pm 0.4) \times 10^{-4} \mathrm{fm}^{3}[5]$.

\subsection{Spin Polarizabilities}

For interactions at increasingly higher energies, the response of the nucleon spin to an electric or magnetic field begins to contribute through

$$
\begin{aligned}
H_{\mathrm{eff}}^{(3)}=-4 \pi[ & \frac{1}{2} \gamma_{E 1 E 1} \vec{\sigma} \cdot(\vec{E} \times \dot{\vec{E}})+\frac{1}{2} \gamma_{M 1 M 1} \vec{\sigma} \cdot(\vec{H} \times \dot{\vec{H}}) \\
& \left.-\gamma_{M 1 E 2} E_{i j} \sigma_{i} H_{j}+\gamma_{E 1 M 2} H_{i j} \sigma_{i} E_{j}\right]
\end{aligned}
$$

The four $\gamma$ factors, called the spin polarizabilities, depict the response to an excitation by an electric or magnetic dipole (E1 or M1) or quadrupole (E2 or M2) followed by a de-excitation by an electric or magnetic dipole. Unlike the scalar polarizabilities, there had been no experimental determination of the values, other than several linear combinations of them such as the forward spin polarizability

$$
\gamma_{0}=-\gamma_{E 1 E 1}-\gamma_{E 1 M 2}-\gamma_{M 1 E 2}-\gamma_{M 1 M 1},
$$

for which $\gamma_{0}=(-1.0 \pm 0.08) \times 10^{-4} \mathrm{fm}^{4}[6][7]$, and the backward spin polarizability

$$
\gamma_{\pi}=-\gamma_{E 1 E 1}-\gamma_{E 1 M 2}+\gamma_{M 1 E 2}+\gamma_{M 1 M 1},
$$

for which $\gamma_{\pi}=(8.0 \pm 1.8) \times 10^{-4} \mathrm{fm}^{4}$ [8]. With the use of these two relations, one can change to a basis of $\gamma_{E 1 E 1}$, $\gamma_{M 1 M 1}, \gamma_{0}$, and $\gamma_{\pi}$, the use of which will be demonstrated in Sec. 4.

Though limited in experimental numbers, there have been various theoretical calculations and predictions of the spin polarizabilities, as given in Table 1. To address this

Table 1. Spin polarizabilities, in units of $10^{-4} \mathrm{fm}^{4}$, K-matrix calculation [9], dispersion relation calculations HDPV [10] and DPV [11][12], chiral Lagrangian calculation $L_{\chi}$ [13], heavy baryon and covariant chiral perturbation theory calculations $\mathrm{HB} \chi \mathrm{PT}[3]$ and $\mathrm{B} \chi \mathrm{PT}[14]$.

\begin{tabular}{|c|c|c|c|c|c|c|}
\hline & K-mat. & HDPV & DPV & $L_{\chi}$ & HB $\chi$ PT & B $\chi$ PT \\
\hline$\gamma_{E 1 E 1}$ & -4.8 & -4.3 & -3.8 & -3.7 & $-1.1 \pm 1.8$ & -3.3 \\
\hline$\gamma_{M 1 M 1}$ & 3.5 & 2.9 & 2.9 & 2.5 & $2.2 \pm 0.7^{1}$ & 3.0 \\
\hline$\gamma_{E 1 M 2}$ & -1.8 & -0.02 & 0.5 & 1.2 & $-0.4 \pm 0.4$ & 0.2 \\
\hline$\gamma_{M 1 E 2}$ & 1.1 & 2.2 & 1.6 & 1.2 & $1.9 \pm 0.4$ & 1.1 \\
\hline$\gamma_{0}$ & 2.0 & -0.8 & -1.1 & -1.2 & -2.6 & -1.0 \\
\hline$\gamma_{\pi}$ & 11.2 & 9.4 & 7.8 & 6.1 & 5.6 & 7.2 \\
\hline
\end{tabular}

need for experimentally extracted spin polarizabilities, as well as improved scalar polarizabilities, the A2 collaboration at the Mainz Microtron (MAMI) facility, located in Mainz, Germany, began a program of Compton scattering experiments to investigate the proton polarizabilities. This

\footnotetext{
${ }^{1}$ Note in additional to the theory error given, there's an additional error on $\gamma_{M 1 M 1}$ of 0.5 , as described in the paper [3].
}

program utilized polarized photon beams with both polarized and unpolarized proton targets to measure the unpolarized cross section as well as three asymmetries

$$
\Sigma_{2 x / z}=\frac{N_{x / z}^{R}-N_{x / z}^{L}}{P_{\gamma} P_{T}\left(N_{x / z}^{R}+N_{x / z}^{L}\right)}, \quad \Sigma_{3}=\frac{N_{\|}-N_{\perp}}{P_{\gamma}\left(N_{\|}+N_{\perp}\right)} .
$$

The first two of these asymmetries rely on a circularly polarized photon beam and a transversely or longitudinally polarized proton target, respectively, where $N_{x / z}^{R / L}$ represents the number of events with $x / z$ target polarization and $R / L$ beam helicity, $P_{\gamma}$ represents the magnitude of the beam polarization, and $P_{T}$ represents the magnitude of the target polarization. The third relies on a linearly polarized photon beam on an unpolarized proton target, where $N_{\| / \perp}$ represents the number of events with $\| / \perp$ beam polarization, and $P_{\gamma}$ represents the magnitude of the beam polarization.

\section{Experiment}

\subsection{Beam}

The MAMI facility is a cascade of racetrack microtrons which can accelerate electrons up to energies of $1.6 \mathrm{GeV}$ [15], with the additional capability of polarizing the beam longitudinally to a high degree [16]. The A2 hall is a real photon facility in which the electron beam strikes a thin radiator, producing Bremsstrahlung photons. The choice of a diamond radiator produces linearly polarized photons through coherent Bremsstrahlung, whereas an amorphous radiator combined with a longitudinally polarized electron beam produces circularly polarized photons through a helicity transfer process. The residual electron is bent in a large spectrometer magnet into an array of 352 scintillators, permitting the tagging of the photon and determination of its energy with a resolution dependent upon the electron beam energy [17]. The photon beam is then collimated before intersecting with the target.

\subsection{Targets}

For measuring $d \sigma_{0} / d \omega$ and $\Sigma_{3}$, an unpolarized liquid hydrogen target is sufficient. For $\Sigma_{2 x}$ and $\Sigma_{2 z}$, however, a target of polarized protons is required. This is accomplished with a Frozen Spin Target (FST) [18] through the process of Dynamic Nuclear Polarization [19]. The process begins by cooling the target to approximately $0.2 \mathrm{~K}$ and using a large dipole magnet to align the electron spins. The polarization is then transferred to the proton by injecting microwaves at the appropriate frequency to cause spin-flip transitions. Once the desired polarization is achieved, the target is further cooled to about $25 \mathrm{mK}$ in order to 'freeze' the spins in place. Even at this temperature, the target would still depolarize in a few hours without a field. To make room for the detectors, however, the polarizing magnet needs to be removed, at which point a weaker holding coil is energized to maintain the polarization. The Mainz FST has been online since 2009, achieving polarizations up to $90 \%$, with relaxation times of over $1000 \mathrm{~h}$. 
Free radicals are needed in the FST in order for it to polarize, which necessitates the target material to be composed of more than just hydrogen. While several options are available, the Mainz group has opted to work with butanol, $\mathrm{C}_{4} \mathrm{H}_{9} \mathrm{OH}$. This does complicate the analysis due to the inclusion of carbon and oxygen in the target, as well as the helium bath used to cool it down. A special carbon target was manufactured such that it could be inserted into the same cryostat, and run as a separate beamtime, to subtract out the contribution of the non-hydrogen nuclei in the butanol target.

\subsection{Detectors}

To analyze the final state of the interaction, a nearly $4 \pi$ detector system is put in place over the target. This detector system is composed of the Crystal Ball (CB) [20] and Two Arms Photon Spectrometer (TAPS) [21] systems. The CB is an array of $672 \mathrm{NaI}$ crystals, covering $20^{\circ}<\theta<160^{\circ}$ and $-180^{\circ}<\phi<180^{\circ}$. This is coupled with a barrel of 24 plastic scintillators called the Particle Identification Detector (PID), as well as a pair of Multi-Wire Proportional Chambers (MWPC), for charged particle identification and tracking [22]. TAPS is a vertical wall of 384 $\mathrm{BaF}_{2}$ and $72 \mathrm{PbWO}_{4}$ crystals, which covers the downstream hole of the CB. It has an additional wall of plastic scintillators for charged particle identification. This system is shown in Fig. 2.

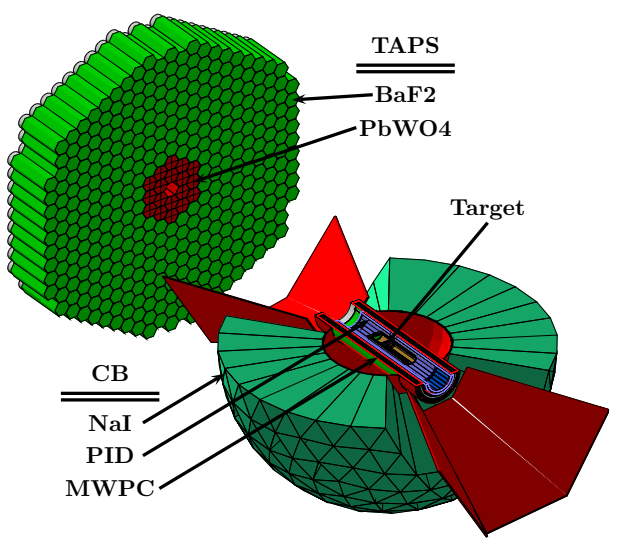

Figure 2. Combined CB/TAPS detector system.

\section{Analysis}

A Compton scattering event is identified in the analysis by selecting final states where a single neutral track and a single charged track are detected. Coincidence between the time of the neutral track and the time of a hit in the tagger allows for tagging the initial state photon. In a high flux environment, however, the tagger experiences a large amount of accidental coincidences, for which a sideband subtraction is necessary, as shown in Fig. 3. The charged track time is not utilized in the accidental subtraction due

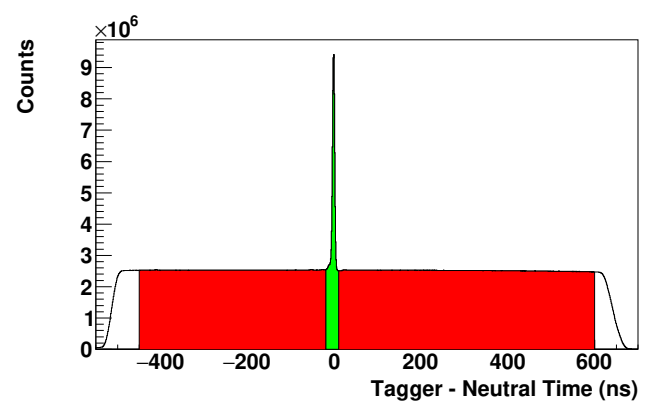

Figure 3. Timing difference between a neutral track and a hit in the Tagger. The sharp 'prompt' peak at zero sits on a large accidental background which is removed by integrating two sideband regions, shown in red, and subtracting it from the integration of the prompt region, shown in green, after scaling by the ratio of the widths of these regions.

to the Time of Flight (TOF) experienced by the heavier proton. While the distance from the target center to the center of a NaI crystal in the CB is only $45 \mathrm{~cm}$, TAPS is three to four times that distance from the target center, depending on the inclusion of a Cherenkov detector. Protons detected in TAPS, a large fraction of the phase space in this analysis, therefore exhibit a notable TOF. As noted in Sec 1, the spin polarizabilities play larger roles at higher energies; unfortunately extraction of these parameters at higher energies is more problematic, notably as more reaction channels open up. A compromise of measuring just below the two-pion photoproduction threshold ( $308 \mathrm{MeV})$ was taken, and an additional cut is made on tagger channels within the region of interest.

Assuming an incoming photon with four-momentum $q_{i}=\left(E_{i}, \vec{p}_{i}\right)$ strikes an at rest proton $k_{i}=\left(m_{p}, 0,0,0\right)$ and results in a scattered photon $q_{f}=\left(E_{f}, \vec{p}_{f}\right)$, the 'missing' four-momentum is then $k_{f}=q_{i}+k_{i}-q_{f}$, and the missing mass for this reaction can then be calculated via

$$
m_{m i s s}=\sqrt{\left(E_{i}+m_{p}-E_{f}\right)^{2}-\left(\vec{p}_{i}-\vec{p}_{f}\right)^{2}},
$$

under which $m_{\text {miss }}=m_{p}$ for a Compton scattering event. While the detection of the proton is required, energy losses suffered by it before detection prevent a total missing energy calculation. These losses will be discussed in Sec. 4.4. Instead, the vector of the track is compared to the vector of $k_{f}$, and an opening angle cut on this greatly reduces background, notably from coherent and incoherent scattering on carbon, oxygen, and helium. Any remaining background from these reactions is then removed by subtracting out the separate data with the carbon target mentioned in Sec. 2.2.

Given that neutral pion ( $\pi^{0}$ photoproduction has a cross section about 100 times larger than Compton scattering in the $\Delta(1232)$ region, an appreciable background signal is generated by cases where one of the decay photons $\left(\pi^{0} \rightarrow \gamma \gamma\right)$ goes undetected, and in some kinematic regions the reconstruction can survive the opening angle cut. This background can be studied either through a careful sim- 
ulation, or directly through the data itself. Similar to the sideband analysis of the tagger accidentals, one can take neutral pion events, which are fully detected in the system, where one of the decay photons is close to the known holes in the detector phase space. By neglecting this photon and analyzing the other decay photon, and the recoil proton, as if they were a Compton scattering event, one can mimic the background in the missing mass distribution. This distribution, along with the various backgrounds, is shown in Fig. 4.

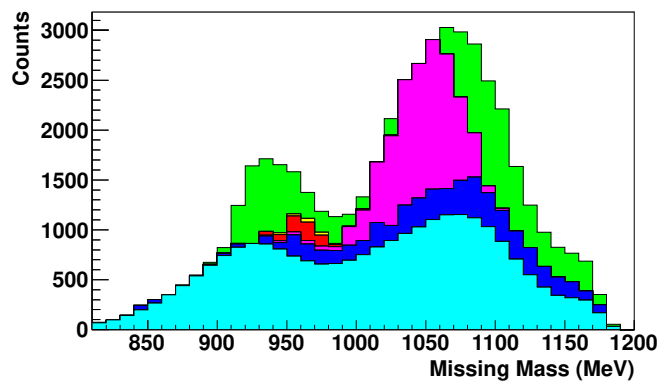

Figure 4. Missing mass distribution showing the tagger accidental (light blue), carbon (dark blue), and neutral pion (magenta, red, and yellow) background contributions, leaving the Compton signal in green.

Subtracting out the various background contributions results in the distribution shown in Fig. 5. Missing mass distributions like this one are then integrated up to conservative values near the proton mass, to ensure that any remaining neutral pion background is not included.

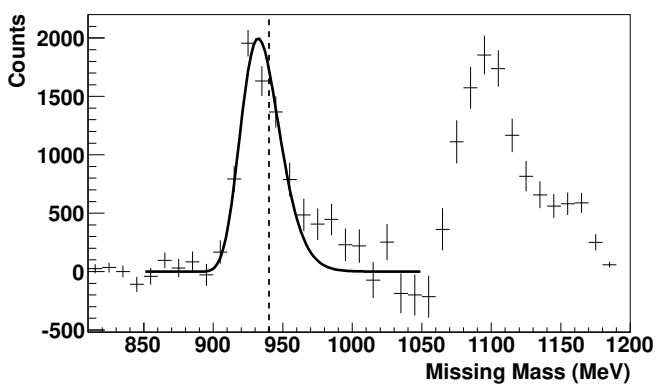

Figure 5. Missing mass distribution after background subtraction, including a fit of the simulated line-shape.

\section{Results}

The A2 collaboration has completed its initial program of Compton scattering experiments with the goal of extracting the spin polarizabilities. The results, in some cases preliminary, are given below for each of the sub-projects.

\section{1 $\Sigma_{2 x}$ : Transverse Target, Circular Beam}

Measuring $\Sigma_{2 x}$ required the use of the polarized target, with the polarization direction transverse to the incoming beam of circularly polarized photons. The asymmetry was then constructed either by flipping the helicity of the photon beam, or by flipping the polarization of the target. While it takes roughly a day to flip the direction of the target polarization, the beam helicity is flipped regularly at a rate of about $1 \mathrm{~Hz}$. This eliminates potential systematic errors due to a change in detection efficiency between positive and negative target polarization data. However, to check such systematics, the target polarization was additionally flipped, albeit only once or twice during a beamtime. The missing mass distributions for each helicitypolarization combination were integrated up to the proton mass, and then applied into Eq. 7, to produce the points shown in Fig. 6. This reflects data in the energy range of $E_{\gamma}=273-303 \mathrm{MeV}$ [23].

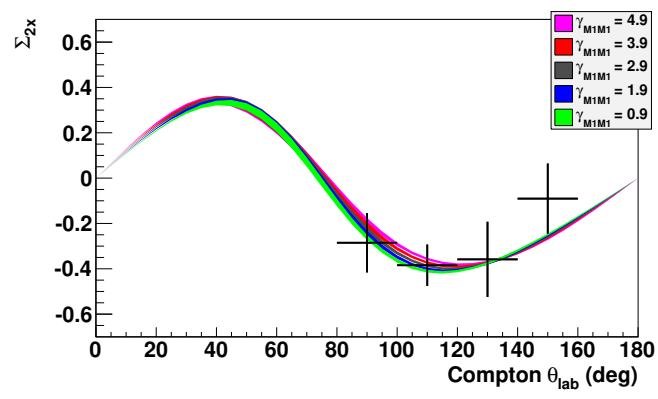

Figure 6. $\Sigma_{2 x}$ for $E_{\gamma}=273-303 \mathrm{MeV}$. The theoretical curves are from the HDPV dispersion code using different values for $\gamma_{M 1 M 1}$ as differentiated by color, while $\alpha_{E 1}$ and $\beta_{M 1}$ are assigned the PDG2012 values, $\gamma_{0}=-1.0, \gamma_{\pi}=8.0$, and $\gamma_{E 1 E 1}=-4.3$. The width of each band is generated by allowing $\alpha_{E 1}, \beta_{M 1}, \gamma_{0}$, and $\gamma_{\pi}$ to vary by their experimental errors [23].

The curves shown in this figure were generated with the use of a dispersion relation code, provided by B. Pasquini [12]. The 2012 PDG numbers [4] were used for the initial scalar polarizabilities values, and the HDPV numbers [10], as shown in Tab. 1, were used for the initial spin polarizability values. In Fig. 6, the initial value for $\gamma_{M 1 M 1}$ was then perturbed by \pm 1 and \pm 2 , in the standard units, producing the different colored lines. The spread of each line reflects an additional step of allowing $\alpha_{E 1}$, $\beta_{M 1}, \gamma_{0}$, and $\gamma_{\pi}$, to vary by their experimental errors, while keeping $\gamma_{E 1 E 1}$ fixed, and summing the resulting shifts in quadrature. What Fig. 6 then depicts is a rough idea of the sensitivity of this asymmetry to $\gamma_{M 1 M 1}$, which is clearly small. Fig. 7 was constructed in a similar way except for varying $\gamma_{E 1 E 1}$ and keeping $\gamma_{M 1 M 1}$ fixed instead. Here one can see a much greater sensitivity to this particular spin polarizability. With just this one observable, one can extract values of $\gamma_{E 1 E 1}=-4.6 \pm 1.6$ and $\gamma_{M 1 M 1}=-7 \pm 11$ [23]. Obviously the value for $\gamma_{M 1 M 1}$ is poorly fit, as predicted by the sensitivity curves, and both values are constrained 


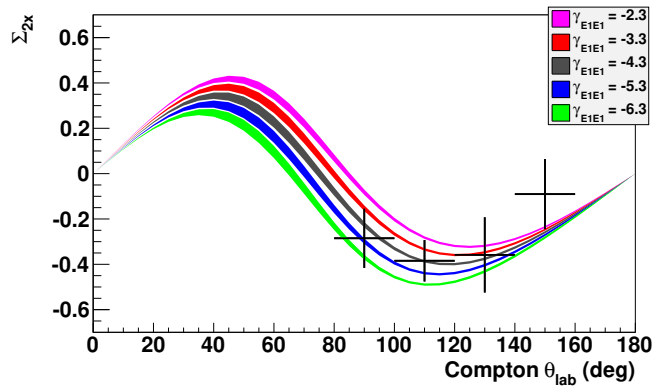

Figure 7. $\Sigma_{2 x}$ for $E_{\gamma}=273-303 \mathrm{MeV}$. Similar to Fig. 6, except now $\gamma_{E 1 E 1}$ is allowed to vary, while one fixes $\gamma_{M 1 M 1}=2.9$ [23]

in the fit by the four other polarizabilities. For these reasons, more data on different observables was required.

\section{2 $\Sigma_{3}$ : Unpolarized Target, Linear Beam}

While measuring $\Sigma_{3}$ only required the use of the simpler unpolarized $\mathrm{LH}_{2}$ target, it then required the use of a diamond radiator to generate a linearly polarized photon beam. The plane of polarization is adjustable, and was flipped by $90^{\circ}$ roughly each hour. Instead of constructing the asymmetry by the events with each beam helicity, it was then determined by the polarization plane. The asymmetry was generated as a function of $\phi$ in addition to $E_{\gamma}$ and $\theta$. The distribution over $\phi$ was fit with a $\cos (2 \phi)$ function, with the amplitude then representing the magnitude of the asymmetry at that $E_{\gamma}$ and $\theta$. This was done for two different energy ranges, $E_{\gamma}=267.0-287.2 \mathrm{MeV}$ and $E_{\gamma}=286.9-307.1 \mathrm{MeV}$, the latter of which is shown in Figs. 8 and 9, with similar sensitivity curves as in Sec. 4.1.

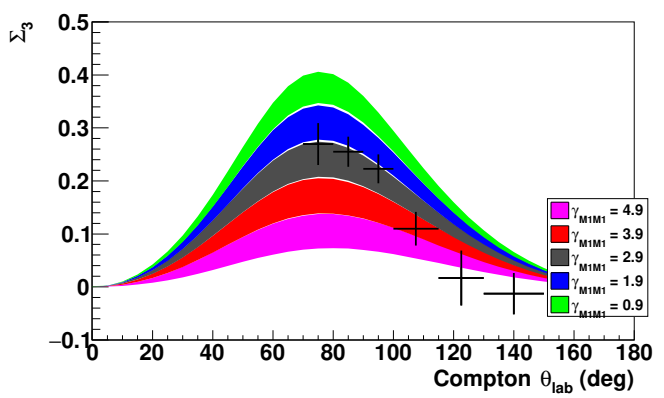

Figure 8. Preliminary $\Sigma_{3}$ for $E_{\gamma}=286.9-307.1 \mathrm{MeV}$. Similar to Fig. 6.

These results obtained at MAMI [24] complement those from a previous experiment at LEGS [25]. Each set of $\Sigma_{3}$ data can then be taken with the $\Sigma_{2 x}$ data, from which the polarizabilities can be fit while still utilizing the four constraints. The results of this inquiry are given in Tab. 2 . The results from both MAMI and LEGS, for both energy ranges, are shown in Figs. 10 and 11. The theoretical

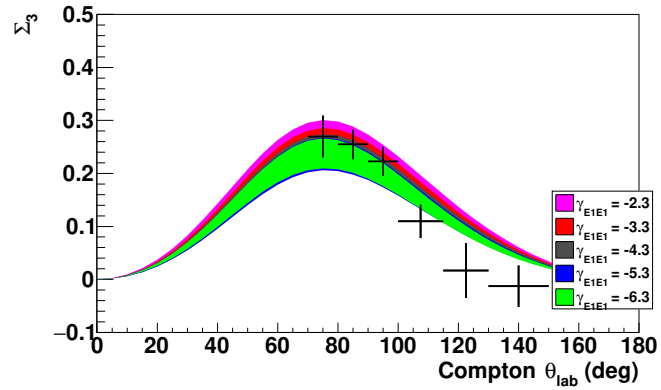

Figure 9. Preliminary $\Sigma_{3}$ for $E_{\gamma}=286.9-307.1 \mathrm{MeV}$. Similar to Fig. 7.

Table 2. Dispersion relation fitted to $\Sigma_{2 x}$ along with either $\Sigma_{3}^{\text {MAMI }}$ [24] or $\Sigma_{3}^{\text {LEGS }}$ [25]. Scalar polarizabilities in units of $10^{-4} \mathrm{fm}^{3}$, spin polarizabilities in units of $10^{-4} \mathrm{fm}^{4}$.

\begin{tabular}{|c|c|c|}
\hline & $\Sigma_{2 x}$ and $\Sigma_{3}^{\text {LEGS }}$ & $\Sigma_{2 x}$ and $\Sigma_{3}^{\mathrm{MAMI}}$ \\
\hline \hline $\bar{\gamma}_{E 1 E 1}$ & $-3.5 \pm 1.2$ & $-5.0 \pm 1.5$ \\
$\bar{\gamma}_{M 1 M 1}$ & $3.16 \pm 0.85$ & $3.13 \pm 0.88$ \\
$\bar{\gamma}_{E 1 M 2}$ & $-0.7 \pm 1.2$ & $1.7 \pm 1.7$ \\
$\bar{\gamma}_{M 1 E 2}$ & $1.99 \pm 0.29$ & $1.26 \pm 0.43$ \\
\hline$\gamma_{0}$ & $-1.03 \pm 0.18$ & $-1.00 \pm 0.18$ \\
$\gamma_{\pi}$ & $9.3 \pm 1.6$ & $7.8 \pm 1.8$ \\
$\bar{\alpha}+\bar{\beta}$ & $14.0 \pm 0.4$ & $13.8 \pm 0.4$ \\
$\bar{\alpha}-\bar{\beta}$ & $7.4 \pm 0.9$ & $6.6 \pm 0.7$ \\
\hline$\chi^{2} /$ dof & 1.05 & 1.25 \\
\hline
\end{tabular}

curves using the values given by the respective fits are also shown in Figs. 10 and 11. Additionally, the theoretical

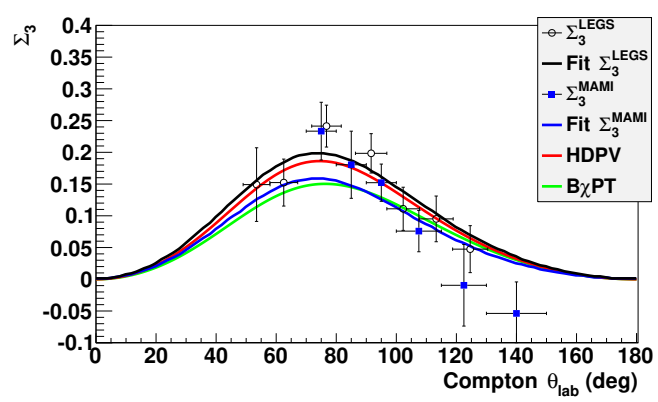

Figure 10. Preliminary $\Sigma_{3}$ for $E_{\gamma}=267.0-287.2 \mathrm{MeV}$. The open circle data are those taken at MAMI [24], while the closed square data are those from LEGS [25]. The black and blue curves represent fitting the $\Sigma_{2 x}$ data from MAMI along with the $\Sigma_{3}$ data from either MAMI or LEGS, respectively, as shown in Tab. 2. The red and green curves show the theoretical predictions from $\mathrm{HDPV}$ and $\mathrm{B} \chi \mathrm{PT}$, respectively.

curves from the HDPV and $\mathrm{B} \chi \mathrm{PT}$ codes, using their values from Tab. 1, are plotted in those figures, depicting a better relationship between the HDPV theory and the fit- 


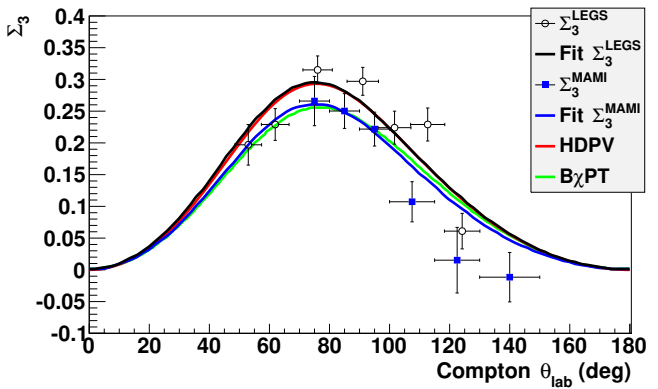

Figure 11. Preliminary $\Sigma_{3}$ for $E_{\gamma}=286.9-307.1 \mathrm{MeV}$. Similar to Fig. 10.

ted values from $\Sigma_{3}^{\text {LEGS }}$ [25], and between the B $\chi$ PT theory and the fitted values from $\Sigma_{3}^{\text {MAMI }}$ [24].

\section{3 $\Sigma_{2 z}$ : Longitudinal Target, Circular Beam}

Completing the picture, and aiding the extraction of the spin polarizabilities without the use of the $\gamma_{0}$ and $\gamma_{\pi}$ constraints, requires an additional lever-arm. This was accomplished in the form of $\Sigma_{2 z}$, another polarized target asymmetry. In this case, the polarization of the target is set longitudinally with respect to the incoming beam of circularly polarized photons. These data are the most recent taken in this A2 Compton program, and as such are still in the process of being analyzed. Some preliminary asymmetry values are shown in Figs. 12 and 13, also for the energy range $E_{\gamma}=273-303 \mathrm{MeV}$. They only include about half of the data taken, were analyzed with only rough calibrations applied, and only use some average polarization values. As such, these will certainly change upon completion of the analyses of Paudyal and Rajabi. However, from the preliminary values one can at least observe both reasonable agreement with the curves as well as indications of reasonably high constraining power of these data.

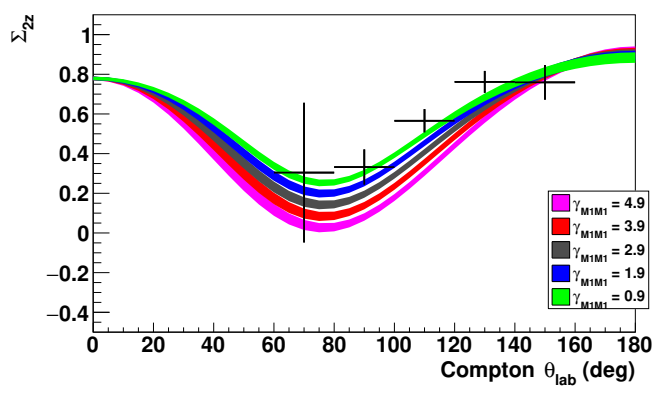

Figure 12. Preliminary $\Sigma_{2 z}$ for $E_{\gamma}=273-303 \mathrm{MeV}$. Similar to Fig. 6 .

\subsection{Active Target}

As noted in Sec. 3, these analyses suffer in the detection of the recoil proton to the degree that its energy can not

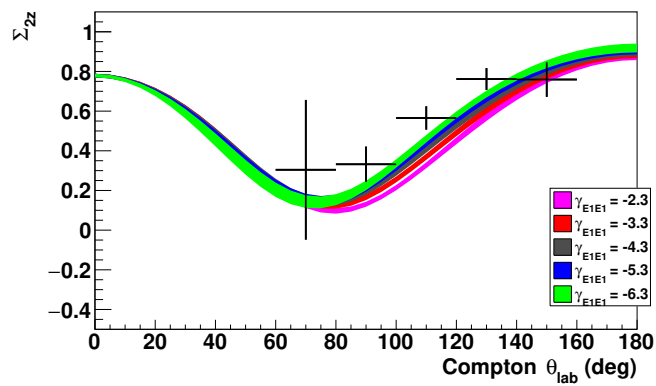

Figure 13. Preliminary $\Sigma_{2 z}$ for $E_{\gamma}=273-303 \mathrm{MeV}$. Similar to Fig. 7.

be absolutely determined. The energy and angular phase space is also limited by the energy loss of the proton, as lower energy recoils will simply not be detected. This can be verified by determining the proton efficiency from $\pi^{0}$ photoproduction, as shown in Fig. 14. This shows that the

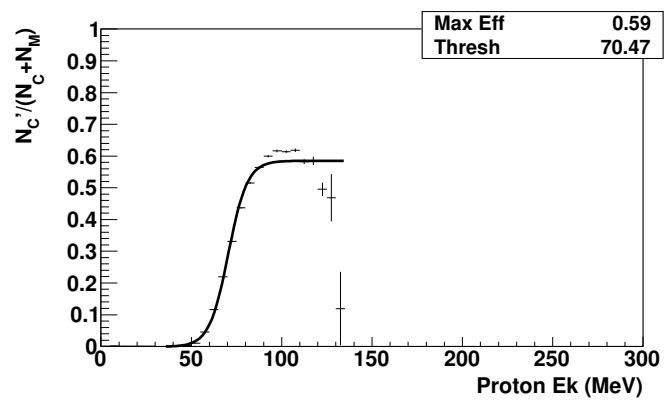

Figure 14. Proton efficiency $\epsilon=N_{C}^{\prime} /\left(N_{C}+N_{M}\right)$ for the frozen spin target, where $N_{C}^{\prime}$ is the number of reconstructed $\gamma p \rightarrow \pi^{0} p$ events that have a charged particle which satisfies an opening angle cut, $N_{C}$ is the number of events that have a charged particle, and $N_{M}$ is the number of events that missed the recoil particle.

threshold for detection in the FST, determined at the halfmax of the distribution, is approximately $70 \mathrm{MeV}$, and by $50 \mathrm{MeV}$ the efficiency is basically zero. The recoil proton energy for the Compton scattering phase space is shown in Fig. 15. With a threshold of $70 \mathrm{MeV}$, only the region in the upper right hand corner is accessible. For this reason it is difficult to go below $270 \mathrm{MeV}$ or forward of $80^{\circ}$. The case is only slightly improved for the $\mathrm{LH}_{2}$ target, where the threshold is about $50 \mathrm{MeV}$. To reach much lower energies, however, requires a different approach.

For this purpose an active polarized target has been developed, composed of discs of scintillating, yet polarizable, polystyrene as schematically shown in Fig. 16. When a charged particle passes through the material, it produces scintillation light. This light is then propagated along the plane of each disc and captured by the target nose, which then propagates it down the length of a light guide tube before reaching a ring of Silicon Photomultipliers (SiPMs). Since these SiPMs can detect single photons, this target 


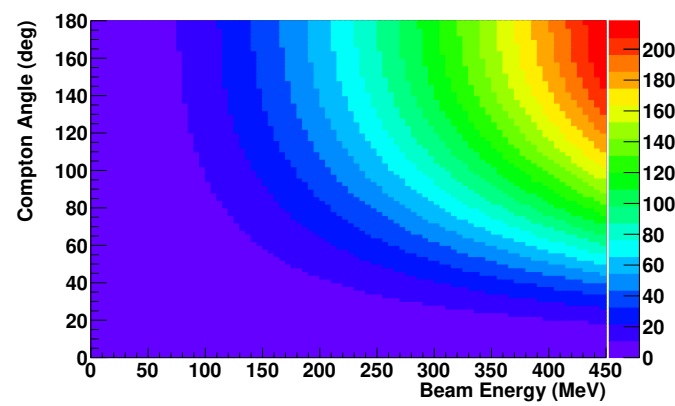

Figure 15. Compton kinematics, showing the recoil proton energy as color gradients as a function of the Compton scattering angle and the incoming beam energy.

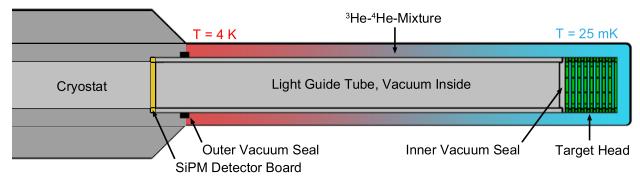

Figure 16. Schematic of the active target [26].

can detect recoil protons immediately after the reaction, but before they have lost energy traversing the target material, cryostat, and various other sections before reaching the main detector system. Such a system will enable analyses at much lower energies, especially around the $\pi^{0}$ photoproduction threshold. One can already analyze the $\mathrm{LH}_{2}$ target data in this region as, both below and slightly above threshold, other reactions can be kinematically separated from Compton scattering. With the polarized target however, as described in Sec. 3, the inclusion of non-hydrogen nuclei complicate things. With the detection ability of the active target, the dominant coherent background from these nuclei can be eliminated as the entire nucleus recoils, rather than individual protons. A first run with the active target, in a transversely polarized state, was performed in 2016, and the data is in the process of being analyzed. Two major achievements of this run are already evident however, the first being the detection of the scintillations within the target, and the second being the polarization of the target. This latter was clear from the NMR readings during polarization, but also confirmed in the analysis by looking at a simple $\pi^{0}$ target asymmetry, as shown in Fig. 17. The clear flip in the asymmetry between target polarization states proves the target was indeed polarized. The asymmetry is small since the analysis had not factored in the actual value of the target polarization, or removed contributions from the carbon which act to dilute the asymmetry.

\subsection{Scalar Polarizabilities}

In addition to studying the spin polarizabilities of the proton, the A2 collaboration undertook to improve the situa-

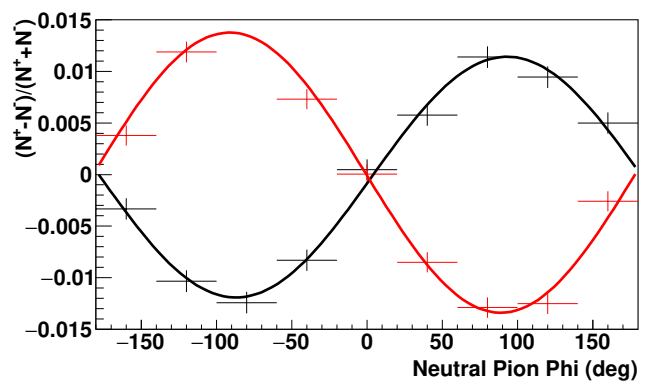

Figure 17. Preliminary $\pi^{0}$ target asymmetry from the active target, with the black points from the positively polarized target and the red points from the negatively polarized target, along with fits to the respective data.

tion on the scalar polarizabilities. Initial studies, also of the $\Sigma_{3}$ observable with an $\mathrm{LH}_{2}$ target, although at incident photon energies below the $\pi^{0}$ photoproduction threshold have been recently published [27]. As noted in Sec. 4.4, Compton scattering events from $\mathrm{LH}_{2}$ can still be analyzed at these energies, despite the absence of recoil proton detection. These asymmetries are shown in Fig. 18, as presented in the paper [27].

With these data, fits were performed in the $\mathrm{B} \chi \mathrm{PT}$ [2][29][14] and $\mathrm{HB} \chi \mathrm{PT}$ [3] frameworks, which led to magnetic polarizability values of $\beta_{M 1}=2.8_{-2.1}^{+2.3} \times$ $10^{-4} \mathrm{fm}^{3}$ and $\beta_{M 1}=3.7_{-2.3}^{+2.5} \times 10^{-4} \mathrm{fm}^{3}$, respectively [27]. These data represent a proof-of-principle run taken in June 2013. A longer run, with improvements in both the tagging system and the linear beam polarization stability, is planned for the second half of 2017. This run will provide both a reduction in the asymmetry errors by a factor of about 3.5, and a set of cross section measurements, that together will enable a single extraction of $\alpha_{E 1}$ and $\beta_{M 1}$ at level of the PDG errors.

\section{Conclusions}

The Compton scattering program at MAMI has included experiments on transversely and longitudinally polarized targets with a circularly polarized photon beam, as well as on an unpolarized liquid hydrogen target with a linearly polarized beam, with the intention of extracting the proton polarizabilities. Much of the data has already been acquired, and the analyses of these data are almost completed. From those analyses that are already completed, preliminary extractions of the scalar and spin polarizabilities have been performed, with the results presented here. The first run with a transversely polarized active target took place in 2016, and results are eagerly anticipated given the observed success of both the scintillation and polarization components of the target. A future run to extend the scalar polarizability study is in the preparation stage, and upon completion of these analyses, an unconstrained fit of the full data set will yield the first definitive determination of these important nucleon structure terms. 


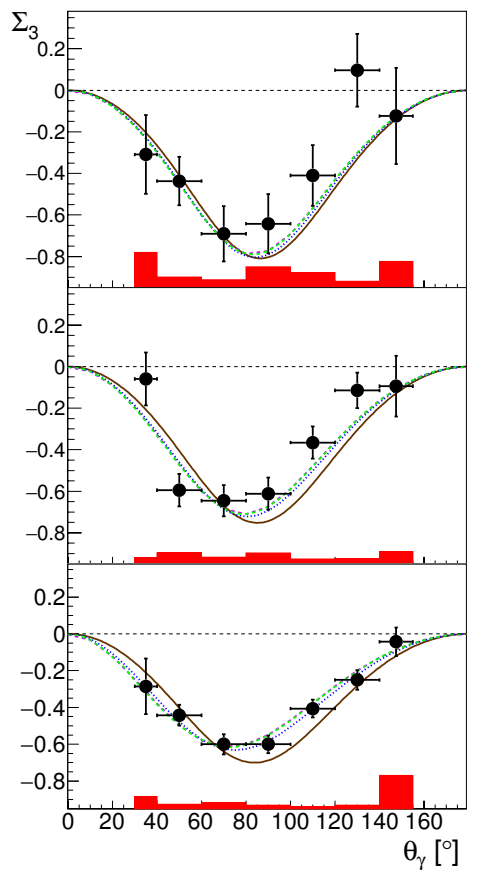

Figure 18. Beam asymmetry $\Sigma_{3}$ for three energy ranges (uppermost: $79-98 \mathrm{MeV}$, middle: $98-119 \mathrm{MeV}$, lowermost: 119 $139 \mathrm{MeV}$ ). The errors represent statistical errors, the red bars indicate the systematic error. Green dashed curve: $\mathrm{B} \chi \mathrm{PT}$ calculation [2], magenta dashed-dotted: DR calculation [28][11], blue dotted: $\mathrm{HB} \chi \mathrm{PT}$ [3], all with $\alpha_{E 1}=10.65 \times 10^{-4} \mathrm{fm}^{3}$ and $\beta_{M 1}=3.15 \times 10^{-4} \mathrm{fm}^{3}$; brown solid: Born term (curves correspond to the central values of the shown energy bins) [27].

\section{Acknowledgments}

We wish to thank the accelerator group and operators of MAMI for their outstanding support; H.W. Grießhammer, J.A. McGovern, V. Pascalutsa, B. Pasquini, and D.R. Phillips for theory support; and the Messina conference organizers for the talk invitation.

\section{References}

[1] V. Olmos de Leon et al., Eur. Phys. J. A 10, 207 (2001).

[2] V. Lensky and V. Pascalutsa, Eur. Phys. J. C 65, 195 (2010).

[3] J.A. McGovern, D.R. Phillips, H.W. Grießhammer, Eur. Phys. J. A 49, 12 (2013).

[4] J. Beringer et al. (Particle Data Group), Phys. Rev. D 86, 010001 (2012).
[5] C. Patrignani et al. (Particle Data Group), Chin. Phys. C 40, 100001 (2016).

[6] J. Ahrens et al. (GDH/A2), Phys. Rev. Lett. 87, 022003 (2001).

[7] H. Dutz, K. Helbing, J. Krimmer, T. Speckner, and G. Zeitler et al. (GDH), Phys. Rev. Lett. 91, 192001 (2003).

[8] M. Camen et al. (A2), Phys. Rev. C 65, 032202 (2002).

[9] S. Kondratyuk and O. Scholten, Phys. Rev. C 64, 024005 (2001).

[10] B.R. Holstein, D. Drechsel, P. Pasquini, and M. Vanderhaeghen, Phys. Rev. C 61, 034316 (2000).

[11] B. Pasquini, D. Drechsel, M. Vanderhaeghen, Phys. Rev. C 76, 015203 (2007).

[12] D. Drechsel, B. Pasquini, and M. Vanderhaeghen, Phys. Rep. 378, 99 (2003).

[13] A.M. Gasparyan, M.F.M. Lutz, and B. Pasquini, Nucl. Phys. A 866, 79 (2011).

[14] V. Lensky and J.A. McGovern, Phys. Rev. C 89, 032202 (2014).

[15] K.-H. Kaiser et al., Nucl. Instrum. Methods Phys. Res., Sect. A 593, 159 (2008).

[16] V. Tioukine, K. Aulenbacher, and E. Riehn, Rev. Sci. Instrum. 82, 033303 (2011).

[17] J. McGeorge, J. Kellie, et al., Eur. Phys. J. A 37, 129 (2008).

[18] A. Thomas, Eur. Phys. J. Special Topics 198, 171 (2011)

[19] D. G. Crabb and W. Meyer, Annu. Rev. Nucl. Part. Sci. 47, 67 (1997).

[20] A. Starostin et al. (CB), Phys. Rev. C 64, 055205 (2001).

[21] R. Novotny, IEEE Trans. Nucl. Sci. 38, 379 (1991).

[22] C. M. Tarbert et al. (CB@MAMI/A2), Phys. Rev. Lett. 100, 132301 (2008).

[23] P.P. Martel, R. Miskimen, et al. (A2), Phys. Rev. Lett. 114, 112501 (2015).

[24] C. Collicott, Ph.D. thesis, Dalhousie University (2015).

[25] G. Blanpied et al. (LEGS), Phys. Rev. C 64, 025203 (2001)

[26] M. Biroth, P. Achenbach, E. Downie, and A. Thomas (A2), PoS PSTP 2015, 005 (2015).

[27] V. Sokhoyan, E.J. Downie, E. Mornacchi, J.A. McGovern, N. Krupina, et al. (A2), Eur. Phys. J. A 53, 14 (2017).

[28] D. Drechsel, M. Gorchtein, B. Pasquini, M. Vanderhaeghen, Phys. Rev. C 61, 015204 (1999).

[29] V. Lensky, J.A. McGovern, V. Pascalutsa, Eur. Phys. J. C 75, 604 (2015). 